Questions vives

\section{Questions Vives}

Recherches en éducation

Vol.9 $n^{\circ} 19$ | 2013

Éducation routière, changement de comportement et formation à la conduite : constat, enjeux et transformations

\title{
Thème, histoire et problématique en éducation à la sécurité routière
}

Theme, history and issues in teaching road safety

Line Courteaud et Christian Roux

\section{(2) OpenEdition}

Journals

Édition électronique

URL : http://journals.openedition.org/questionsvives/1300

DOI : 10.4000/questionsvives. 1300

ISBN : 978-2-8218-1394-6

ISSN : $1775-433 X$

Éditeur

Université Aix-Marseille (AMU)

Édition imprimée

Date de publication : 15 juillet 2013

Pagination : 141-157

ISBN : 978-2-912643-43-8

ISSN : 1635-4079

Référence électronique

Line Courteaud et Christian Roux, «Thème, histoire et problématique en éducation à la sécurité routière », Questions Vives [En ligne], Vol. 9 n $^{\circ} 19$ | 2013, mis en ligne le 15 octobre 2013, consulté le 30 avril 2019. URL : http://journals.openedition.org/questionsvives/1300 ; DOI : 10.4000/ questionsvives. 1300

Ce document a été généré automatiquement le 30 avril 2019.

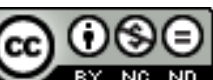

Questions Vives est mis à disposition selon les termes de la licence Creative Commons Attribution Pas d'Utilisation Commerciale - Pas de Modification 4.0 International. 


\title{
Thème, histoire et problématique en éducation à la sécurité routière
}

Theme, history and issues in teaching road safety

\author{
Line Courteaud et Christian Roux
}

\section{Introduction}

1 Cet article entre dans le cadre d'une recherche dans le champ de l'Éducation à la Sécurité routière, recherche entreprise dès 2011 sous la forme d'un master recherche deuxième année en sciences humaines et sociales - mention sciences de l'éducation, et prolongée sous la forme d'une thèse de doctorat dont le thème porte sur le processus d'autoévaluation dans l'apprentissage de la conduite. Dans le chapitre conclusif de cette première recherche clinique relative à l'apprentissage authentique ${ }^{1}$ au sein de la formation du conducteur, la prise de conscience des valeurs humaines ainsi que l'intersubjectivité dans l'espace routier ont émergé comme éléments concourant à la sécurité proactive des usagers de la route. En effet, les théories humanistes privilégiant les notions de « soi » de « liberté » et « d'autonomie », selon Rogers (2005) incitent à « un changement dans la conduite de l'individu» (p. 189). Dans ce contexte « la conduite», qui sous-tend une façon de se comporter ou de se conduire, met en évidence l'aspect psychologique de la conduite. Le choix de cette recherche s'appuie sur les principaux rapports ${ }^{2}$ en matière de sécurité routière, lesquels soulignent la capacité auto-évaluative comme dynamique de changement dans les attitudes et comportements du conducteur. Ce travail qui s'inscrit dans le paradigme de la pensée complexe, a pour cadres théoriques: les théories de l'apprentissage ; l'auto-évaluation; la pluridisciplinarité ; le triptyque de Michel Foucault : voir, surveiller, punir... et s'appuie méthodologiquement sur une approche clinique de l'activité . 


\section{Contexte}

2 Depuis le premier permis de conduire ${ }^{4}(1889)$, la réussite à cet examen a longtemps été tributaire d'un "savoir-faire » : démarrer, s'arrêter, diriger le véhicule et avoir quelques notions de dépannage. Pourtant, la maîtrise du véhicule n'évite pas l'accident. Or, depuis quelques décennies, la recherche en matière de sécurité routière ayant mis en lumière le «système ": homme-véhicule-environnement, a permis d'aborder l'insécurité routière selon une approche plus systémique. La complexité du système routier ne permet plus de penser la conduite automobile seulement sous l'angle des automatismes élémentaires pour le maniement du véhicule. Au travers de cette vision plus globale, les études cliniques $^{5}$ d'accidents ainsi que les analyses comportementales du conducteur ont fait apparaitre l'intervention de ce dernier comme élément régulateur - ou pas ! - au sein de l'interaction du système. La sécurité ne relève donc pas seulement de la performance, mais aussi de l'attitude manifestée par le comportement du conducteur. Apparaît ainsi une nouvelle dimension : celle du «savoir-être » ou «savoir se conduire ». Récemment, cette nouvelle dimension a été intégrée à l'examen du permis de conduire, les Inspecteurs dudit permis ayant pu bénéficier d'une formation adaptée. Mais ce ne fut pas le cas pour les enseignants de la conduite; par conséquent, leurs pratiques se révèlent être en inadéquation avec les nouvelles exigences de l'examen. En effet, l'apprentissage du "savoir-être » implique des pratiques autres que celles mises en place pour l'acquisition des habiletés psychomotrices ${ }^{6}$. Cette absence de formation continue au sein de la formation du conducteur, bien que ce soit un enjeu sociétal majeur, témoigne des difficultés de reconnaissance que rencontre cette profession.

\section{Problématique}

Malgré l'évolution technologique ${ }^{7}$ des véhicules, l'environnement routier demeure un espace insécure. Le permis de conduire ne devrait-il pas être un gage de sécurité ? La formation du conducteur ne serait-elle plus en adéquation avec le permis de conduire et les conditions actuelles de circulation? En matière d'insécurité routière, quelle est la politique gouvernementale ? Éducative ou répressive?

\section{Démonstration}

\section{Automobile et comportement}

Malgré les dangers générés par l'automobile, cette technologie a su s'imposer comme principal mode de déplacement dans les pays développés ${ }^{8}$. Or, dès son apparition, l'automobile fut repérée comme à l'origine de conflits sur la route. Mais, l'automobile, en tant que telle, n'y est pour rien, c'est le comportement du conducteur qui est en cause. Les psychiatres du début du XIX siècle décrivaient le comportement du conducteur comme « l'ultime étape d'une forme spéciale de folie » (Bailet, 1999, p. 154). La littérature de la Belle Epoque ${ }^{9}$ faisait écho d'une angoisse de la population, qui « s'accélérait avec la naissance de l'automobile » (p. 153). André Dumont ${ }^{10}$, brossant l'état d'esprit des citoyens disait «Hier soir à six heures, j'ai failli être écrasé avec ma femme et mes enfants, par trois messieurs, montés dans une automobile lancée à la vitesse d'une locomotive» 
(Bailet, 1999, p. 153). En 1927, à la tribune de l'Assemblée Nationale, le député Lambert traitait les automobilistes de "têtes de fous [...] d'aliénés qui se tuent avec une férocité gaie » (p. 154). Il soulignait aussi le comportement à risque de l'automobiliste qui prenait la liberté de traverser les « agglomérations en ouragan, les dépassements à droite à cent à l'heure » (p. 154). Incontestablement, dans cet environnement dynamique, l'homme motorisé ne savait plus contrôler ses impulsions et s'en trouvait submergé.

\section{L'homme et la dimension sociale de la conduite}

5 Avec la démocratisation ${ }^{11}$ de l'automobile survenue en France après la première guerre mondiale, la voiture devint un symbole de liberté individuelle offrant à l'homme la possibilité de se déplacer rapidement et de façon autonome. Elle apparait comme un véritable raccourci du temps, modifiant les rapports spatio-temporels de l'individu. Grâce à la vitesse ${ }^{12}$, l'homme est en mesure de dépasser ses limites physiologiques; en même temps, va se développer chez lui un sentiment de domination et de toute puissance. Par ailleurs, au travers de la typologie du véhicule, le conducteur a la possibilité d'exprimer son rang et sa réussite sociale. La voiture est donc vécue comme le prolongement de l'habitat, véritable sphère privée, dans laquelle le conducteur se sent protégé et à l'abri des pressions extérieures. Ainsi tout "écart» intempestif est considéré comme une agression à la propriété privée, et le conducteur exacerbé choisit d'extérioriser ce débordement très souvent par un comportement agressif et incivique. Cette manière d'être manifeste l'attitude habituelle ou circonstanciée du conducteur. L'attitude est déterminée par la situation de conduite qui se présente. L'attitude est porteuse de sens, sinon d'intentions, ce qui a permis de mettre en évidence les différents comportements routiers : à risque et sécuritaire.

\section{Le système routier : l'évolution du véhicule}

Depuis son invention ${ }^{13}$ en 1769 , l'automobile ${ }^{14}$ a connu une évolution rapide grâce aux progrès technologiques. C'est ainsi que dès le milieu du XIX ${ }^{e}$ siècle, l'énergie à vapeur ${ }^{15}$ fut remplacée par le pétrole grâce à l'invention du moteur à explosion ${ }^{16}$ et du carburateur. Et c'est la découverte du cycle thermodynamique ${ }^{17}$ à 4 temps ${ }^{18}$ qui permit au moteur à combustion interne de s'imposer à partir de 1886. C'est aussi à partir de ce moteur que Karl Benz fabriqua la première automobile (tricycle) aboutie, équipée d'une boite de vitesse, d'un différentiel, et pouvant rouler à la vitesse moyenne de $15 \mathrm{~km} / \mathrm{h}$. Actuellement, ce même moteur, bénéficiant d'une haute technologie allie performance, sécurité et économie. En effet, l'injection électronique qui améliore la combustion, permet de diminuer la consommation de carburant et par conséquent réduit le rejet de gaz à effet de serre. En outre, l'électronique embarquée ${ }^{19}$ fournit aussi une assistance aux fonctions du véhicule et du conducteur, par exemple : l'antiblocage des roues, qui est une fonction d'aide à la conduite et qui neutralise l'action inadaptée du conducteur, tout en se substituant à lui, afin d'assurer un freinage efficace pour sauvegarder la sécurité du véhicule, des passagers, et de l'environnement. Mais, tout système ayant ses limites, une prise de risque trop importante (prendre un virage trop vite par exemple) de la part du conducteur ne peut éviter la sortie de route du véhicule. Donc, sans accompagnement éducatif, cette nouvelle technologie, pourrait s'avérer contre-productive pour la sécurité. Par ailleurs, les équipements routiers, qui ont eux aussi bénéficié du développement technologique, tendent à garantir une protection maximale aux usagers de la route. 


\section{L'évolution de l'infrastructure}

7 Actuellement, le réseau routier est géré selon le type de voie ${ }^{20}$ par l'État, les collectivités territoriales et les sociétés concessionnaires. Ce sont les Romains ${ }^{21}$ à l'époque du Premier Empire qui ont mis en place environ $12000 \mathrm{~km}$ de voies pavées entre Rome et les frontières, afin de pouvoir déplacer facilement les armées, alors que le reste du territoire était parcouru par des chemins de terre. Avec la chute de l'Empire, ces voies furent dégradées et au Moyen Age, il ne restait plus que des routes régionales et des chemins plus ou moins en bon état. Au XVIII siècle, avec le concours du ministère de la guerre, le service des ponts et chaussées établit un véritable réseau routier dans le but d'unifier le pays et faciliter le passage des troupes. Après la révolution de 1789, Napoléon remplaça les routes royales par des routes impériales et développa particulièrement l'accès vers l'Italie. Le décret du 16 décembre 1811 va distinguer trois classes de routes : la première classe partait de Paris et aujourd'hui, les principales routes nationales ${ }^{22}$ dérivent de cette classe. Ainsi, la RN7 qui commence au point zéro des routes de France, sur le parvis de la cathédrale de Notre Dame de Paris, part en direction du Sud, d'où son nom historique de «route bleu ou route des vacances» et se prolonge par la route italienne ${ }^{23}$ pour se terminer à Rome; la seconde classe se composait des routes reliant les grandes villes de provinces entre elles; la troisième classe correspond aux routes départementales actuelles. Les chemins vicinau ${ }^{24}$ ont, quant à eux, été créés par la loi du 28 juillet 1824. Mais, l'automobile supportant moins bien les irrégularités de la chaussée que les véhicules hippomobiles, l'État dut prendre des directives. C'est ainsi, qu'entre les deux guerres les routes nationales et départementales furent goudronnées ${ }^{25}$. En parallèle, de grands chantiers furent mis en œuvre, dont l'autoroute ${ }^{26}$ reconnue d'utilité publique en 1935. Le premier tronçon entre Saint-Cloud et Orgeval $(20 \mathrm{~km})$ fut mis en service le 9 juin 1946. La première société d'autoroute, Estérel Côte d'Azur (ESCOTA) fut créé en 1956 avec pour objectif la réalisation de l'autoroute $\mathrm{A} 8^{27}$, afin de désengorger la RN7 dans une région aux reliefs tourmentés. Le 16 juillet 1965, le Général de Gaulle et Saragat, le Président italien, inauguraient le tunnel du Mont-Blanc ${ }^{28}$ lequel allait favoriser la mobilité et le déplacement international. Il se produit alors un phénomène de circularité, le développement de l'infrastructure entraînant le développement de l'automobile, et viseet-versa. De nos jours, le réseau routier est pensé de façon à réduire aussi bien le risque d'accident que la gravité de celui-ci. C'est ainsi que les routes à double sens de circulation ont été remplacées par les routes à sens unique. Le réseau autoroutier, reconnu comme le moins accidentogène, devrait à long terme prédominer sur le territoire national. L'infrastructure est donc régie par une politique de sécurité routière au même titre que la réduction des dangers induits par l'environnement.

\section{L'environnement}

8 L'environnement est un espace où interagissent le facteur humain et la dynamique des véhicules. Il a donc fallu trouver un moyen de communications et d'échanges approprié. Après la première guerre mondiale la signalisation ${ }^{29}$ fut adoptée comme outil cohérent de communication. Toutefois, ce principe de codification semble insuffisant pour assurer l'intersubjectivité de ce milieu, car incontestablement, la présence humaine déclenche l'incident ou l'accident. C'est ainsi, qu'entre 1990 et 2006, les arbres $^{30}$ en bordure des route furent abattus, dans le but de protéger les vies humaines lors des sorties de route 
du véhicule. Depuis, la mise en place des glissières de sécurité a permis de sauvegarder les $a_{r b r e s}{ }^{31}$ au détriment de la sécurité des motocyclistes qui lors de chute vont s'encastrer entre les rails et la chaussée. Ces éléments ont donc été modifiés ou améliorés de façon à assurer la sécurité de tous les usagers. L'environnement semble fonctionner comme un écosystème puisque « chaque activité se réalisant dans l'écosystème a pour contrepartie une réponse. Chaque interaction, chaque échange, si minime soit-il, représentent potentiellement un mécanisme de régulation. L'ensemble de ces mécanismes maintient la communauté dans un équilibre dynamique » (De Rosnay, 1975. p. 32).

\section{Règlementation et comportement}

9 Le décret du 10 mars 1899 instaure le véritable Code de la route selon les fondements juridiques de la loi du 30 mai 1851 concernant la «police de roulage et des messageries publiques ", dont la préoccupation était essentiellement la protection des chaussées se creusant d'ornières. Ce décret réglementa la vitesse à $20 \mathrm{~km} / \mathrm{h}$ en agglomération et 30 $\mathrm{km} / \mathrm{h}$ en rase-campagne; la carte grise ${ }^{32}$ fut instituée. Mais, devant l'ampleur du phénomène automobile, une commission fut chargée d'élaborer un Code de la route ; c'est ainsi qu'en 1922 fut adopté un ensemble de dispositions législatives et règlementaires régissant la circulation sur la voie publique. L'ordonnance du 15 décembre $1958^{33} \mathrm{mit}$ l'accent sur le comportement du conducteur et particulièrement sur ses attitudes à risques. Depuis, le Code de la route a été régulièrement adapté à la prise de conscience de la vitesse et de la consommation l'alcool comme facteur de risques. Ce sont ces «facteurs de risques » que les pouvoirs publics tentent d'enrayer.

\section{L'insécurité et la politique de sécurité routière}

Entre 1960 et 1971, le nombre de tués sur les routes, passant de 8000 à 15000 morts par an, décida les pouvoirs publics à mettre en place une politique de prévention et de sécurité. En 1971, le Service National des Examens du Permis de Conduire (SNEPC) et le Comité Interministériel à la Sécurité Routière (CISR) ${ }^{34}$ furent crées. Le CISR du 17 décembre 1993 inscrivit la formation du conducteur comme axe majeure dans le renforcement de la sécurité routière. Mais en 2002, les derniers chiffres de l'observatoire National Interministériel de la sécurité Routière (ONISER) dénombraient encore plus de 7000 morts sur les routes. Ces piètres résultats engagèrent Nicolas Sarkozy, ministre de l'Intérieur, dans une politique de lutte contre l'insécurité routière. Un budget fut mis à disposition d'objectifs ambitieux, tels le développement d'une culture générale de prévention des risques, le durcissement de la politique pénale et une meilleure sécurité des infrastructures et des véhicules. En parallèle, des mesures répressives furent mises en place, dont la multiplication des équipements ${ }^{35}$ automatiques visant à améliorer la détection et le traitement des infractions. Depuis le déploiement de cette batterie de radars, l'infraction à la vitesse a rétrogradé en deuxième position derrière l'alcool et le nombre de tués ${ }^{36}$ sur les routes n'a cessé de décroitre. Incontestablement, la sanctionrépression qui est utilisée à la fois pour changer les comportements et faire respecter la règle est un moyen de lutte efficace contre l'insécurité routière. Alors qu'il détient le permis de conduire qui le rend apte à circuler dans l'espace public , l'homme motorisé irrespectueux des règles choisit de subir cette dictature mise en place sous forme de contrôle pouvant mener à la répression. 


\section{Le permis de conduire : autorisation administrative}

Devant l'ampleur du phénomène automobile, le Préfet de police, Louis Lépine, institua par l'ordonnance du 14 août 1893, un «certificat de capacité » obligatoire pour circuler dans le département de la Seine. Reconnu comme l'ancêtre du permis de conduire ce certificat était délivré par la préfecture sur rapport d'un ingénieur des Mines, expert accrédité pour contrôler le savoir technique des candidats masculins âgés de plus de 21 ans. La vitesse était alors limitée à $12 \mathrm{~km} / \mathrm{h}$ en agglomération et $20 \mathrm{~km} / \mathrm{h}$ en campagne. En 1897, la Duchesse d'Uzès est la première femme à obtenir le certificat de capacité. En 1922, avec la refonte du Code de la route, le permis de conduire remplaça le certificat de capacité ; différentes catégories ${ }^{37}$ furent instituées, l'âge minimal fut abaissé à 18 ans, alors que le savoir-faire était toujours contrôlé par un expert accrédité par le Ministre des travaux publics. En 1954, la distinction des catégories de permis fut effectuée par des lettres alphabétiques (A, B, C, D, E, F). Depuis, seule la catégorie F (conducteurs infirmes) a été supprimée, les progrès technologie ayant permis l'aménagement du véhicule en fonction du handicap; tandis que les autres catégories ${ }^{38}$ n'ont connu des réformes que relatives aux équivalences ${ }^{39}$. Mais, depuis 2006, en vue d'harmoniser les règles relatives au permis de conduire, l'Union européenne adopta une nouvelle règlementation, instaurant de nouvelles catégories ${ }^{40}$ principalement pour la conduite des véhicules à deux roues et les véhicules de transport de personnes ou de marchandises. L'entrée en vigueur de ces mesures, au 19 janvier 2013, renforce le principe de l'accès progressif des conducteurs à certains véhicules, du fait de leur puissance, de leur poids, et de l'utilisation qui en est attendue. Ces changements entrent dans le cadre d'une politique globale de sécurité, dans laquelle le permis de conduire est une garantie, dès lors que l'annulation du permis est susceptible de mettre hors circuit le conducteur à risque ou dangereux. En outre, grâce à la mise en place du permis à points ${ }^{41}$ cette dimension réductrice a évolué vers une dimension éducative. En effet, l'infraction n'étant plus seulement considérée sous l'angle de la répression, puisque le conducteur, ayant commis une infraction entraînant un retrait de points, aura la possibilité de les racheter en suivant une formation ${ }^{42}$ de sensibilisation à la sécurité routière; de fait, cette mesure incite l'ensemble des conducteurs à adopter un comportement responsable.

Le $1^{\text {er }}$ mars 2004, l'entrée en vigueur du permis probatoire va renforcer le permis à points. C'est un permis progressif qui s'adresse au conducteur débutant. Doté d'un capital initial de 6 points, le conducteur aura la possibilité de récupérer les 6 autres sur une période de 2 ou 3 ans, à condition de ne commettre aucune infraction. En revanche, la perte de trois points ou plus impose une formation de sensibilisation à la sécurité routière. Cette mesure post-permis qui permet la prise d'expérience tend à réduire l'implication des jeunes de 18/24 ans dans les accidents mortels de la circulation. Au-delà du droit administratif de conduire, le permis véhicule des valeurs, telles, la liberté et l'autonomie. En effet, le permis est considéré comme un rite de passage vers l'âge adulte, et aussi un passeport pour l'insertion sociale et professionnelle; ce qui explique sa popularitée ${ }^{43}$. Mais comment se décline l'examen du permis de conduire? 


\section{L'épreuve du permis de conduire}

13 Lorsque Léon Serpollet se présenta à l'examen, en 1889, l'ingénieur des mines ${ }^{44}$ contrôla son savoir (notions de dépannage) et savoir-faire technique (démarrer, s'arrêter, diriger le véhicule). Depuis toutes les réformes ont modifié la forme et non le fond. Avant 1972, il était possible d'échouer à l'épreuve théorique tout en réussissant l'épreuve pratique. Dès lors, le candidat devait repasser uniquement la partie manquante. À cette époque, une connaissance parfaite des règles du code de la route était nécessaire pour répondre aux questions orales posées par l'expert. Or, depuis 1972, une refonte de l'examen du permis de conduire sépara l'épreuve théorique de la pratique. Il fallait obtenir l'examen théorique avant de pouvoir passer l'épreuve pratique. Depuis, l'évaluation théorique générale (E.T.G) des connaissances est réalisée par le biais d'un questionnaire à choix multiple. Selon une méthode audiovisuelle, 40 scènes de conduite se succèdent et le candidat doit être capable de répondre sans erreur à 35 d'entre elles. La réforme ${ }^{45} \mathrm{de} 2010$ introduisit dans l'épreuve théorique l'analyse des situations de conduite. C'est une avancée qui permit de mettre en lumière le rôle des attitudes dans l'activité de conduite, tandis que l'évaluation sommative finale du simple décompte d'erreurs fut remplacée par une évaluation des compétences ${ }^{46}$. L'évaluation du savoir-faire vise les connaissances, les habiletés psychomotrices et la maîtrise des situations de conduite; tandis que celle du savoir-être sera à même d'apprécier le comportement adopté vis-à-vis du partage de la route avec les autres usagers, la courtoisie au volant ainsi que la conduite économique et respectueuse de l'environnement. Enfin, l'évaluation du risque sera en mesure de déterminer la prise de conscience de celui-ci par le biais d'un comportement autonome et sûr. Quel est donc l'impact de cet examen sur la formation du conducteur?

\section{La formation du conducteur}

\section{Organisation}

14 En 1917, lorsque l'auto-école apparait, le moniteur doit être titulaire d'une carte professionnelle, qui fut remplacée, par décret du 15 décembre 1958, par le Certificat d'Aptitude Professionnelle et Pédagogique (CAPP). Cette réforme permit à la corporation de se professionnaliser et de s'orienter vers une logique en rapport avec la sécurité routière. Depuis, les réformes n'ont cessé de se succéder; le décret du 02 Août 1979, remplaça le CAPP par le Certificat d'Aptitude pour l'Enseignement de la Conduite des véhicules terrestre à moteur (CAPEC), lui-même remplacé par le décret du 30 novembre 1986 qui institua le Brevet pour l'Exercice de la Profession d'Enseignant de la Conduite et de la Sécurité Routière (BPECASER). Avec cette réforme, l'appellation « enseignant de la conduite » se substitua à celle de «moniteur d'auto-école ». La formation du conducteur allait alors connaitre un profond bouleversement et s'enrichir dans ses pratiques enseignantes.

\section{Programme}

En 1989, avec la mise en place du Programme National de Formation ${ }^{47}$ (PNF), l'enseignant pu disposer d'un outil pour appréhender les éléments concourant à une bonne gestion de la conduite. L'objectif général de ce programme était d'amener le conducteur à la maitrise des connaissances afin de développer les savoir-faire et les attitudes positives vis-à-vis de 
la sécurité routière. Les savoirs et les savoir-faire essentiellement tournés vers la maîtrise du véhicule (habiletés psychomotrices) et l'adaptation aux situations de conduite (tâche de conduite) représentent l'apprentissage type de la conduite, alors que le savoir-être, influencé par les attitudes, sollicite un apprentissage beaucoup plus complexe avec des connaissances d'ordre supérieur : comportement et prise de conscience.

Actuellement, sous la pression économique, la formation initiale privilégie toujours l'acquisition des automatismes élémentaires pour maîtriser le véhicule.

\section{Filières d'apprentissage et pratiques pédagogiques}

17 Avant 1972, le moniteur ne dispensait pas vraiment de cours sur la règlementation, car l'élève se servait du code de la route pour apprendre les règles de circulation. Après 1972, influencée par l'examen du permis de conduire, la formation du conducteur va elle aussi séparer la théorie de la pratique. L'élève attend la réussite à l'examen de code avant de commencer l'apprentissage pratique. Or, cette absence de mise en lien entre théorie et pratique va générer des incompréhensions et amplifier les difficultés au sein de l'apprentissage ${ }^{48}$. Dans la perspective de remplacer cette filière traditionnelle, reconnue peu performante, une nouvelle filière d'apprentissage fut expérimentée à partir de 1984 . C'est ainsi qu'en 1987, l'Apprentissage Anticipé de la Conduite (AAC) ${ }^{49}$, fut généralisé à l'ensemble du territoire national, l'objectif étant de permettre la prise d'expérience par un accès graduel à la conduite et réduire ainsi l'implication des jeunes conducteurs dans les accidents mortels de la circulation. Or, à l'encontre des idées reçues, le retour d'expérience révéla que cette nouvelle filière était autant, sinon, plus impliquée dans les accidents mortels de la circulation. Il semblerait qu'après l'obtention du permis de

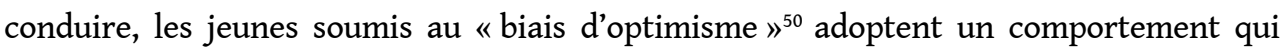
tend à dénier le risque; attitude induisant l'accident. En effet l'activité de conduite qui développe des compétences cognitives et perceptives nécessite aussi la conscience du risque. Les recherches en matière de sécurité routière qui ont permis de mettre en lumière cette approche de la conduite tend à orienter la formation du conducteur dans cette voie.

\section{Les recherches en matière de sécurité routière}

18 Depuis quelques décennies, la recherche en matière de sécurité routière ${ }^{51} \mathrm{~s}^{\prime}$ est articulée autour du système : homme-véhicule-environnement. Cette approche globale a permis à la recherche de bénéficier de progrès dans les domaines de la physiologie, de la psychologie, de l'ergonomie, des sciences de l'éducation et du travail. Dans le domaine de la formation des usagers de la route et du conducteur, le rapport Verré ${ }^{52}$ (1997) juge qu'il " est indispensable d'enrichir la formation initiale par des enseignements relatifs à la psychologie, la psychopédagogie et l'animation de groupe»(p.26). Mais la pression économique qui est de «dépenser le moins possible et obtenir le permis de conduire le plus rapidement possible » (p. 26) contribue à réduire l'apprentissage à « ses dimensions les plus utilitaires, et ce au détriment de sa qualité et de son contenu en termes de sécurité et de comportement apaisé au volant " (p. 26). Tandis que le rapport Lebrun (2008) qui s'est penché sur la modernisation de l'apprentissage de la conduite, renforce l'idée que le permis de conduire est d'avantage "perçu comme l'achat d'un droit de conduire privilégiant le bachotage que comme l'apprentissage progressif d'un savoir-faire avec l'acquisition d'un comportement responsable sur la route»(p. 26). En outre, ce 
dernier rapport met l'accent sur la difficulté de « vouloir concilier acquisition progressive de connaissances, attitudes et reflexes susceptibles de prévenir les comportements à risques, et formation rapide et peu chère » (p. 26). Car, acquérir de l'expérience pour savoir anticiper les risques nécessite du temps. Par ailleurs, ce rapport souligne que le manque d'expérience entraîne le manque de maîtrise, ceci contribuant à augmenter la « sur représentation des jeunes conducteurs dans les accidents liés à des excès de vitesse, à la perte de contrôle du véhicule, ou à des prises de risques ». Le rapport CEESAR (Page, 2004) explique que «l'accident de la route n'est pas toujours la conséquence d'une prise de risque. Il est aussi la conséquence d'une défaillance fonctionnelle que l'on peut trouver dans n'importe quelle autre activité humaine (défaillance dans la chaîne fonctionnelle perception-compréhension-décision-action)» (p. 135). L'état physique (fatigue, sommeil, alcool...) et psychologique (les affects, émotion, joie, surprise, colère...) du conducteur serait à même de provoquer le dysfonctionnement de cette chaîne. Ce dérèglement aurait pour conséquence un traitement cognitif erroné de l'information ce qui occasionnerait une réaction inadaptée à la situation. La conduite automobile n'est donc pas isolée des autres secteurs de la vie.

19 Par ailleurs, lors du quatrième programme-cadre de l'Union Européenne (1994-1998) la France participa au projet de recherche Gadget (Sigrist, 1999) lancé afin d'analyser les effets des systèmes de formation du conducteur sur le comportement et leurs capacités (ainsi que leurs limites) à ouvrir des perspectives. Les résultats de cette recherche ont :

20 - d'une part, souligné l'aspect psychologique de la conduite en présentant une matrice ${ }^{53}$ selon 4 niveaux hiérarchiques du comportement; les niveaux les plus hauts : projets de vie et aptitude à la $v^{5 i}{ }^{54}$, objectif de la conduite automobile et contexte social ${ }^{55}$ influenceraient les niveaux les plus bas: maîtrise des situations de circulation ${ }^{56}$ et maniement du véhicule ${ }^{57}$.

21 - d'autre part, ouvert de nouvelles pistes d'apprentissage, notamment celle de l'autoévaluation et de la gestion du risque. Ainsi, selon ce rapport Gadget : "l'auto-évaluation est une manière d'attirer et de concentrer l'attention sur des lacunes et limites importantes » (p. 30). Ce rapport incite donc la formation du conducteur à favoriser cette capacité auto-évaluative, afin que l'activité de conduite décrite comme « une compétence hiérarchique et comme une compétence de degré élevé, englobant au niveau le plus bas les capacités motrices et au niveau le plus élevé les projets généraux de vie et les aptitudes à la vie » (p. 33) puisse dépasser l'apprentissage des réflexes conditionnés.

Bien que valorisée par de nombreux rapports européens, cette approche complexe de l'apprentissage est encore méconnue du milieu de l'auto-école. Le dispositif, souffrant d'une pénurie en formation continue, utilise toujours des pratiques qui pourtant ne répondent plus ni à la complexité du système routier, ni aux exigences du permis de conduire.

\section{4. Étayage théorique}

\subsection{La pédagogie par objectifs}

Depuis l'expérimentation de l'Apprentissage anticipé de la Conduite (AAC), la Pédagogie Par Objectifs ${ }^{58}$ (PPO) s'est s'imposée comme référentiel théorique dans la formation du conducteur. Or, cette pédagogie qui réduit la formation à une transmission des savoirs 
relatifs aux premiers niveaux taxonomique «limite la créativité en prédéterminant le chemin cognitif» (Torchon, 1990, p. 34). Selon Genthon (1997), cette limite viendrait d'une logique linéaire, qui tend à isoler, enfermer l'apprenant dans une étape, tant que la maîtrise complète des opérations relatives à une tâche n'est pas atteinte. Paradoxalement cette clôture qui "accentue la compréhension et entraîne la généralisation de ces opérations à une même classe de tâches » (Genthon, 1997) entrave la créativité par son manque d'ouverture sur d'autres perspectives, en particulier, l'apparition des transferts d'apprentissage. Or, le transfert qui est une logique qualitative, selon Bonniol, (1981) accompagne le passage de la compréhension à l'extension du critère, en "transformant les critères d'évaluation des tâches scolaires en critères de réussite de tâches scolaires " (p. 440). Ainsi, les critères manipulés dans l'évaluation formative vont glisser vers une nouvelle combinaison pour un autre agencement. Vial (2009) estime, quant à lui, que ce transfert d'apprentissage favorise une prise de conscience nécessaire à tout fonctionnement opératoire, afin d'amener l'apprenant à gérer ses ressources cognitives. La généralisation ainsi que le transfert apparaissent donc comme deux dimensions fondamentales de tout apprentissage, lesquelles vont différencier les visées pédagogiques. La généralisation au service du dispositif visant conformité et production, alors que le transfert ouvert sur la richesse des possibles visera le développement de l'autonomie ${ }^{59}$ par l'activation des processus généraux, tels l'abstraction, la généralisation, la régulation. À l'enseignant de créer les conditions nécessaires pour stimuler et développer ces processus. Dans ce paradigme, l'évaluation s'inscrit comme dynamique de changement. Car, l'enseignant qui adopte la posture de l'évaluateur engage l'apprenant à réfléchir sur sa pratique. Selon Vial (2009) «toute évaluation remplit une fonction réflexive » (p.16) et cultive la dynamique en évitant le stockage du savoir. Cette mise à distance favorise à la fois la problématisation et le questionnement. Or, la formation du conducteur qui est axée sur l'acquisition des automatismes élémentaires pour le maniement du véhicule fait usage de pratiques pédagogiques qui sont inspirées par les théories du conditionnement de Pavlov (1954) et de Skinner (1946), alors que, Delorme (1986) affirme que «les concepts et pratiques behavioristes ne pèsent plus aujourd'hui autant sur la pédagogie par les objectifs ». En matière d'enseignement, la formation du conducteur n'a guère évolué au fil des années, toujours axée sur une forme de behaviorisme, alors qu'il existe bien d'autres modèles théoriques qui seraient plus appropriés à l'apprentissage de la conduite.

\subsection{La pédagogie de l'alternance}

Le modèle de l'alternance dont le dénominateur commun est de vouloir associer théorie et pratique, formation et production semble un référentiel approprié à l'apprentissage de la conduite. L'action engageant à la fois l'intellectuel et le manuel, le passage d'un pôle à l'autre devrait susciter le questionnement et l'analyse des situations vécues, afin d'amener une prise de conscience sur le savoir engrangé, et à partir de l'expérience faire évoluer les représentations ${ }^{60}$. En outre, la pédagogie de l'alternance qui est un modèle valorisant le travail sur la différence "théorie/pratique, solution/problème, école/ entreprise » (Poplimont, 2000, p. 13) tend à favoriser la construction et l'appropriation du savoir par le vécu des situations complexes et la pensée réflexive.

Pour Morin (2005), la complexité (de « complexus : ce qui est tissé ensemble », qui soustend le paradoxe du singulier et du pluriel, décrit aussi un tissu d'évènements, d'actions, 
d'interactions, d'aléas, avec en filigrane l'image de l'inextricable, du désordre, de l'ambiguïté et de l'incertitude. La pensée complexe n'est donc ni linéaire ni simplifiante, mais relève de l'intelligibilité, ce qui permet d'articuler et de relier les éléments du système et non les isoler et les séparer, afin d'en extraire une connaissance multidimensionnelle et non parcellaire.

\subsection{La pensée réflexive}

La pensée réflexive qui procède de l'intelligence est considérée par Guillaumin, Pesce et Denoyel (2009) comme une méthodologie interactive de recherche sous la forme de recherche-action, participative, collaborative et formative, à des fins de compréhension pratique et théorique entrainant l'autonomisation dans l'action. La démarche réflexive peut être développée dans le champ des pratiques professionnelles. Vial (2000) en utilisant le terme "auto-évaluation » l'applique au domaine de la formation. Dans sa vision, l'auto-évaluation relève d'un processus psychosocial qui "se développe et s'organise dans la formation » (p. 7) pouvant « faciliter les échanges entre les partenaires, dans la relation éducative» (p. 7) et ce, à des fins d'émancipation. Pour lui, l'autoévaluation est dépendante de l'évaluation et se décline selon « la logique de l'évaluation valorisée " (p.120); tandis que Bonniol et Vial (2009) la décrivent comme un " prolongement » (p. 214) de l'évaluation formative. Ainsi l'auto-évaluation, qui résulte de la manipulation des critères par l'apprenant et la mise en rapport objectif-critèreopérations à réaliser, va donner à celui-ci le moyen d'analyser ses erreurs. Ce travail réflexif ouvrira sur la mise en œuvre de nouvelles stratégies pour atteindre l'objectif. C'est le principe de l'autocontrôle qui influence la performance du travail en favorisant l'identification des procédures efficaces; la procédure étant reproductible, l'isoler légitime son réinvestissement. Dépendante de l'auto questionnement, cet autocontrôle incite à réfléchir sur l'utilité de ce qui est fait, et qui, selon Vial (2009) demeure en « liens avec l'élaboration du projet personnel » (p. 124). Pour Cardinet (1984) l'analyse par les élèves de leurs perceptions aussitôt après la réalisation d'une activité serait propice aux explications et comparaisons sur leurs façons de faire. Ce qui serait matière à générer la motivation, afin d'améliorer leurs méthodes de travail. Cependant, Dejours (1999) constate que la mobilisation positive de l'homme dans une tâche avec des situations changeantes et imprévues ne peut dans un principe d'efficacité et d'utilité exclure l'échec partiel. En outre, cette démarche réflexive de la pensée, favorisée par le biais du travail de groupe, pourrait servir les objectifs les plus difficiles à observer, tel le développement des valeurs humaines, (liberté, respect, tolérance), lesquelles prédisposent à l'adoption d'un comportement civique et la prise de conscience du risque. Selon Peretti-Watel (2000), le risque est étroitement lié à la position sociale et au système de valeurs de l'individu. Le risque est un construit et « représente le danger librement accepté et individuellement évitable (p.55). Introduire la gestion du risque dans l'apprentissage de la conduite consisterait à travailler sur les causes afin d'en éliminer ou réduire les effets, et ce par une prise de conscience qui amène à comprendre que le risque perçu n'est pas le risque réel. Dans cette optique, la formation du conducteur serait à même de lutter contre l'insécurité routière en déconstruisant les comportements latents à risques et en reconstruisant par le biais d'une éducation au civisme, la considération et l'empathie, afin que l'espace routier devienne un espace apaisé et sécurisé. 


\section{CONCLUSION}

L'apprentissage des automatismes élémentaires semble insuffisant pour assurer la sécurité des usagers de la route, alors que l'apprentissage complexe qui sollicite questionnement et réflexion serait à même de développer la prise de conscience et l'autonomisation dans l'action. Cette prise de conscience serait susceptible de permettre l'accès à une culture de l'automobile en favorisant le passage de l'état de passager et de piéton, à celui de conducteur. Ceci implique une approche globalisante qui tienne compte de l'aspect physiologique, psychologique et sociologique de la conduite. L'aspect physiologique permettrait de cerner les limites humaines et individuelles sur le plan des capacités, tandis que l'aspect psychologique, tourné vers les connaissances sur le développement psychique, tenterait d'appréhender les attitudes et comportements au volant. Enfin, l'aspect sociologique permettrait de saisir le rôle et l'influence de l'automobile sur les relations sociales. De plus, cette approche systémique permettrait à la formation d'ouvrir sur une perspective d'évolution en accord avec les directives européennes en matière de sécurité routière.

Vial, M. (2009). Se former pour évaluer. Bruxelles : De Boeck Université.

\section{BIBLIOGRAPHIE}

Bailet, J-M. (1999). L'Éducation routière. Paris : Que Sais-Je.

Bartl, G., Gregersen, N-P., \& Sanders, N. (2005). Minimum European Requirements for Driving Instructor training. Vienne : Institut Gute Fahrt.

Bonniol, J-J. (1981). Déterminants et mécanismes de comportements d'évaluation d'épreuves scolaires. Thèse de doctorat ès Lettres et Sciences Humaines. Bordeaux II.

Bonniol, J-J., \& Vial, M. (2009). Les modèles de l'évaluation. Bruxelles : De Boeck Université.

Cardinet, J. (1984). Pour apprécier le travail des élèves. Neuchatel : IRDP.

Clot, Y. (2010). La fonction psychologique du travail. (5 $5^{\mathrm{e}}$ éd. corr. $3^{\mathrm{e}}$ tirage). Paris : PUF.

Dejours, C. (1999). Le facteur humain, $2^{\mathrm{e}}$ édition. Paris : PUF.

Delorme, C. (1986). Devenir de la pensée par objectifs en pédagogie. Éducation permanente, $\mathrm{n}^{\circ} 85$. 5-16.

De Rosnay, J. (1975). Le macroscope vers une vision globale. Paris : Seuil.

Genthon, M. (1997). Apprentissage-Évaluation-Recherche. Revue En Question.

Guillaumin, C. Pesce, S., \& Denoyel, N. (2009). Pratiques réflexives en formation. Ingéniosité et ingénieries émergentes. Paris : l'Harmattan.

Hatakka, M., Keskinen, E., Gregersen, N.- P. \& Glad, A. (1999). Théories et objectifs des mesures d'éducation et de formation. In S. Siegrist (Ed.), Formation et évaluation du conducteur, 
obtention du permis de conduire. Vers une gestion théoriquement fondée du risque routier des jeunes conducteurs (pp. 18-50). Résultats du projet européen GADGET, Groupe de travail $n^{\circ} 3$. Berne.

Lebrun, D. (2008). Audit sur la modernisation de l'apprentissage et du permis de conduire. Rapport. Ministère de l'écologie, de l'énergie, du développement durable et de l'aménagement du territoire.

Morin, E. (2005). Introduction à la pensée complexe. Paris : du Seuil.

Page, Y. (2004). Estimation du risque relatif d'implication accidentelle des jeunes conducteurs ayant suivi l'Apprentissage Anticipé de la Conduite. Rapport CEESAR. Fondation MAIF.

Pavlov, I. (1954). Euvres choisis. Moscou : Edition de Moscou.

Peretti-Wattel, P. (2000). Sociologie du risque. Paris : Armand Colin.

Poplimont, C. (2000). Représentations sociales des formateurs dans la formation par alternance. Approche intensive et étude clinique de cas. Thèse de doctorat en sciences de l'éducation. Université de Provence.

Rogers, C. (2005). Le développement de la personne. Paris : Dunod.

Skinner, B. F. (1946). Educational psychology. New York: Prentice All.

Torchon, F.-N. (1990). Didactique du français, de la planification à ses organisateurs cognitifs. Paris :

ESF.

Verré, J. (1997). Formation des usagers de la route et des conducteurs. Rapport. Ministre de l'équipement, des transports et du logement.

Vial, M. (2000). Organiser la formation : le pari sur l'auto-évaluation. Paris : L'Harmattan.

\section{NOTES}

1. Selon le psychothérapeute, C. Rogers, l'apprentissage authentique s'appuyant sur les théories humanistes permet une relation empathique centrée sur autrui, afin d'amener la personne «à se voir elle-même de façon différente ; elle devient plus confiante en elle-même et plus autonome ; elle devient plus souple, moins rigide, dans ses perceptions; elle devient plus acceptante des autres» (2005, p. 189).

2. Rapport GADGET (voir : Hatakka, Keskinen, Gregersen \& Glad, 1999) ; Rapport CEESAR (voir : Page, 2004) ; Projet européen MERIT (voir : Bartl, Gregersen \& Sanders, 2005).

3. Selon le modèle d'Yves Clot (2010) des protagonistes (apprentis-conducteurs) seront associés à une situation de conduite et à son analyse.

4. Léon Serpollet fut le premier candidat à se présenter à l'examen du permis de conduire sur un tricycle à vapeur, qu'il avait lui-même fabriqué.

5. L'Étude détaillée des Accidents (EDA) a été mise en place en France à la fin des années 70 au sein de l'hôpital de Salon-de-Provence, afin de comprendre la genèse et le déroulement de l'accident, ce qui permit de mettre en place des actions préventives efficaces.

6. Maniement et contrôle du véhicule (contrôle du volant, contrôle de la vitesse, freinage, accélération...)

7. Zone de déformation de la carrosserie protégeant l'habitacle en cas de choc, ceinture de sécurité, assistance de direction et de freinage, aérodynamisme, multiplexage, carburation électronique... 
8. En 2010, la densité automobile moyenne en Europe était de l'ordre de 600 véhicules pour 1000 habitants.

9. Période historique de progrès social, économique, technologique et politique en Europe, qui s'étend de la fin du XIX siècle au début de la Première Guerre mondiale.

10. Ingénieur belge (1847/1920) et professeur des Mines / géologie.

11. Survenue grâce au montage à la chaîne qui a été développé dans les années 1900 par le constructeur américain, Henry Ford. Par le biais de cette chaîne d'assemblage mobile l'ouvrier effectue une tâche simple, afin de la réaliser rapidement et parfaitement.

12. En physique, la vitesse est une propriété temporelle associée au déplacement, au changement de lieu, au mouvement.

13. Considéré par les historiens comme la première automobile, le fardier à vapeur inventé par l'ingénieur militaire français, Nicolas-Joseph Cugnot ne servit qu'à l'artillerie pour déplacer de lourds canons. Cet engin était autopropulsé à $4 \mathrm{~km} / \mathrm{h}$, grâce à une chaudière à vapeur ayant une autonomie moyenne de $15 \mathrm{mn}$.

14. En 1861, le terme automobile apparait dans la langue française comme adjectif, formé de auto et de l'adjectif mobile, d'après locomobile, à côté de automoteur, plus ancien. En 1866, il est signalé dans le grand Dictionnaire de Pierre Larousse comme adjectif pour « qui se meut de soimême "; il a un sens voisin de automatique. En 1876, avec l'apparition des moteurs à explosion, le mot prend son essor, et en 1895, l'adjectif est employé à propos de l'activité générée par l'automobile : le sport et l'industrie. Enfin, en 1896, apparait l'abréviation un auto, puis une auto deviendra courante autour de 1900.

15. En 1680, le physicien Isaac Newton, avait émis l'hypothèse que l'expulsion de la vapeur par un tube d'échappement produirait une réaction suffisante pouvant faire avancer un véhicule ; celuici venait d'inventer la propulsion par moteur à réaction.

16. Par l'ingénieur belge Etienne Lenoir, en 1859. Mais du fait que les gaz n'explosent pas mais brûlent avec une vitesse de front de flamme (fine zone dans laquelle se déroule la combustion; elle sépare 2 milieux, un milieu contenant le combustible et l'autre le comburant) inférieure à celle d'une explosion, ce vocable se révéla impropre et fut abandonné et remplacé par celui de « combustion interne ». La combustion étant la transformation rapide d'un carburant et d'un comburant en énergie réalisée grâce à une réaction exothermique.

17. Science de la chaleur et des machines.

18. Mis au point en 1862 par l'ingénieur français, Beau de Rochas (admission, compression, combustion-détente, échappement).

19. C'est un système électronique et informatique autonome, constitué de plus de 100 capteurs qui informent en temps réel plusieurs calculateurs de bord. Il peut être comparé au comportement humain avec une phase d'observation, puis de réflexion et d'action. Ces ordinateurs qui gèrent la quasi-totalité du fonctionnement du véhicule, contrôlent aussi bien la combustion du carburant que la tenue de route et le freinage.

20. Routes nationales : $11800 \mathrm{~km}$; routes départementales : $380000 \mathrm{~km}$; voiries communales : $550000 \mathrm{~km}$; autoroutes non concédées : $1100 \mathrm{~km}$.

21. Domination romaine antique entre 27 av. J.- C. et 476 ap. J.-C.

22. RN1: Paris-Calais ; RN6 : Col du Mont Cenis ; RN7 : longe la côte Méditerranéenne.

23. SS1 : strada statale 1 Via Aurelia.

24. Petites routes de campagne à la charge des communes mais sous le contrôle des préfets.

25. Le goudron est un dérivé houiller de couleur noire.

26. Améliora la sécurité à la fois en désengorgeant le réseau routier et en fluidifiant le trafic, réduisant ainsi les risques routiers; elle participa aussi à développer l'économie (transports routiers).

27. Dite la Provençale, relie Aix-en-Provence et l'A7 à la Côte d'Azur ; longue de $223 \mathrm{~km}$.

28. Dont la convention avait été signée entre les deux pays en 1953. 
29. La signalisation horizontale: marquage au sol; la signalisation verticale: panneaux; la signalisation lumineuse : les feux. Cette signalisation fit l'objet d'une convention internationale signée à Genève le 30 mars 1931; le décret du 03 juillet 1934 autorisa la ratification de cette convention internationale sur l'unification de la signalisation routière (dimensions, formes, couleurs).

30. 6000 arbres ont été abattus dans le Gers.

31. Au XVI ${ }^{e}$ siècle les routes ont été bordées d'arbres afin de délimiter le tracé et de protéger les piétons et les chevaux du soleil.

32. L'idée d'identifier le véhicule date de 1749 ; sous le règne de Louis $\mathrm{XV}$, un officier de la maréchaussée préconisa de doter d'un numéro les véhicules hippomobiles. De nos jours, la carte grisé a été remplacée par l'appellation : « certificat d'immatriculation ». Avant de circuler sur la voie publique, tous les véhicules terrestres à moteur doivent être immatriculés.

33. Sanctionne l'état d'ivresse manifeste relevé chez le conducteur ou sous l'emprise d'un état alcoolique.

34. En 1972, Jacques Chaban-Delmas, alors Premier Ministre, crée le Comité Interministériel à la Sécurité Routière dont la mission est de définir la politique de sécurité routière en France. Il est présidé par le premier ministre et son secrétariat est assuré par le délégué interministériel à la sécurité routière (DISR).

35. Ayant une faible tolérance sur les excès de vitesse ( $5 \mathrm{~km} / \mathrm{h}$ jusqu'à $100 \mathrm{~km} / \mathrm{h}$ et $5 \%$ au-dessus). 36. La norme européenne comptabilise le nombre de tués à 30 jours. Selon cette norme, en 2002, il a été enregistré : 7741 morts et en $2011: 3963$.

37. Tourisme, qui est un tronc commun obligatoire ; motocyclettes ; camions de plus de 3 tonnes ; transport en commun.

38. A : moto ; A1 : motocyclette légère, cylindrée inférieure à $125 \mathrm{~cm}^{3}$. B : véhicule de tourisme dont le poids total autorisé en charge est égal ou inférieur à 3,5 T. C : véhicule de transport de marchandises dont le poids total autorisé en charge est supérieur à 3,5 T. D : véhicule de transport de transport en commun comportant plus de 9 places assises. E (B) : ensembles de véhicules rentrant dans la catégorie $B$, attelés d'une remorque dont le poids total autorisé en charge excède $750 \mathrm{~kg}$ et si le poids total autorisé en charge de la remorque est supérieur au poids à vide du véhicule tracteur ou si la somme des poids totaux autorisés en charge, remorque et véhicule tracteur excède $3500 \mathrm{~kg}$. E (C): ensembles de véhicules couplés rentrant dans la catégorie C, attelés d'une remorque ou d'une semi-remorque. E (D) : ensembles de véhicules couplés rentrant dans la catégorie $\mathrm{D}$, attelés d'une remorque dont le poids total autorisé en charge excède $750 \mathrm{~kg}$.

39. Sous réserves de certaines conditions, le permis B, par exemple, autorise la conduite d'une $125 \mathrm{~cm}^{3}$.

40. AM : âge minimum 14 ans ; types de véhicules : 2 roues ou 3 roues à moteur ; cylindré inférieure ou égale à $50 \mathrm{~cm}^{3}$; Puissance inférieure ou égale à $4 \mathrm{~kW}$; vitesse inférieure ou égale à $45 \mathrm{~km} / \mathrm{h}$. A1 : âge minimum 16 ans ; types de véhicules : motocyclettes $125 \mathrm{~cm}^{3}$ ou tricycles à moteur, avec ou sans side car; puissance inférieure ou égale $11 \mathrm{~kW}$ et rapport puissance/poids inférieur ou égale à $0,1 \mathrm{~kW} / \mathrm{kg}$; tricycles puissance inférieure ou égale à $15 \mathrm{~kW}$. A2 : âge minimum : 18 ans; types de véhicules : motocyclettes avec ou sans side car; puissance inférieure ou égale à $35 \mathrm{~kW}$ et rapport puissance poids inférieure ou égale à $0,2 \mathrm{~kW} / \mathrm{kg}$. $\mathbf{A}$ : âge minimum 24 ans ou 20 ans si A2 depuis au moins 2 ans + formation obligatoire de $7 \mathrm{~h}$; types de véhicules : motocyclettes ou tricycles à moteur avec ou sans sidecar; puissance supérieure à 15 $\mathrm{kW}$. B1 : âge minimum : 16 ans ; types de véhicules : quadricycle à moteur, catégorie L7e ; puissance inférieure ou égale à $15 \mathrm{k} \mathrm{W}$; poids à vide inférieure ou égale à $400 \mathrm{~kg}$ (transport de personne) ou inférieure ou égale à $550 \mathrm{~kg}$ (transport de marchandises). B : âge minimum : 18 ans; types de véhicules : véhicules automobiles comportant 8 passagers (conducteur non compris); Poids Total Autorisé en Charge (P.T.A.C) inférieur ou égal à $3500 \mathrm{~kg}$; remorques: 
poids inférieur ou égal à $750 \mathrm{~kg}$ ou supérieur à $750 \mathrm{~kg}$ et inférieur ou égal à $3500 \mathrm{~kg}$ si Poids Total Roulant Autorisé (P.T.R.A) supérieur à $3500 \mathrm{~kg}$ et inférieur et égal à $4250 \mathrm{~kg}$ avec formation de $7 \mathrm{~h}$. BE : âge minimum : 18 ans; types de véhicules : véhicules automobiles et remorques de catégorie $\mathrm{B}+$ remorque/semi-remorque inférieure ou égale à $3500 \mathrm{~kg}$ et P.T.R.A supérieur à 4250 $\mathrm{kg}$; avoir le permis $\mathrm{B}$. C1 : âge minimum: 18 ans; types de véhicules: transport de marchandises ; véhicules : transportant des passagers inférieur ou égal à 8 dont P.T.A.C inférieure ou égale à $7500 \mathrm{~kg}$; remorques : poids inférieur ou égale à $750 \mathrm{~kg}$ (avoir le permis B). C1E : âge minimum 18 ans; types de véhicules: transport de marchandises avec remorques; véhicules catégorie $\mathrm{C} 1$ + remorque/semi-remorque supérieur à $750 \mathrm{~kg}$; véhicules catégorie $\mathrm{B}$ + remorque/semi-remorque supérieur à $3500 \mathrm{~kg}$; P.T.R.A inférieur ou égal à $12000 \mathrm{~kg}$ (avoir le permis (1). C : âge minimum 21 ans; types de véhicules : transport de marchandises; véhicules : transportant 8 passagers au maximum (non compris le conducteur) P.T.A.C supérieur à $7500 \mathrm{~kg}$; remorque inférieur ou égal à $750 \mathrm{~kg}$ (avoir le permis B). CE : transport de marchandises avec remorque; âge minimum 21 ans; types de véhicules: véhicules catégorie C + remorque/semi-remorque supérieur à $750 \mathrm{~kg}$ (avoir le permis C). D1 : transport de voyageurs; âge minimum 21 ans; types de véhicules : véhicules pouvant transporter au moins 16 passagers (conducteur non compris); longueur inférieure ou égale à $8 \mathrm{~m}$; remorque inférieure ou égale à $750 \mathrm{~kg}$; avoir le permis B. D1E : transport de voyageur avec remorque ; âge minimum : 21 ans; types de véhicules : véhicules catégorie D1 + remorque supérieure à $750 \mathrm{~kg}$; avoir le permis D1. D : transport de voyageurs; âge minimum 24 ans; types de véhicules : véhicules pouvant transporter plus de 8 passagers, pouvant être attelé d'une remorque inférieure ou égale à $750 \mathrm{~kg}$; posséder le permis $\mathrm{B}$. DE : transport de voyageurs avec remorque; âge minimum 24 ans; type de véhicules : véhicule catégorie $D+$ remorque supérieure à $750 \mathrm{~kg}$; avoir le permis D.

41. Institué par la loi du 10 Juillet 1989, entré en vigueur le 01 juillet 1992 ; le permis est doté d'un capital initial de 12 points. Par décision de justice, et selon un barème d'infraction le nombre de points retirés sera d'autant plus grand que l'infraction commise sera grave, et ce jusqu'à l'annulation totale des points et par conséquence l'annulation du permis.

42. Stage payant de 2 jours à la charge du contrevenant et qui permet de récupérer au maximum 4 points. Seulement un stage par an est autorisé.

43. En 2010, un million trois cent mille candidats ont été présentés à l'examen, contre neuf cent mille titres délivrés.

44. Le service des mines est une administration créée en 1781 par Necker (Ministre de Louis XVI) pour permettre à l'État royal de mener une politique minière, afin de pouvoir enquêter sur les mines du royaume sans avoir recours aux ingénieurs du privé. C'est un service technique dont l'action dans le domaine de l'aménagement et de l'industrie exprime l'autorité qu'exerce l'État. Selon la loi du 21 avril 1810 et le décret du 10 novembre 1810, la compétence du service des mines s'étend principalement sur les mines, minières et carrières ainsi que sur les usines sidérurgiques. A ces missions spécifiques, s'ajoutent des compétences, comme le contrôle des machines à vapeur ou la surveillance de l'exploitation des chemins de fer.

45. Par l'arrêté du 19 février 2010.

46. Les experts en ingénierie des ressources humaine définissent la compétence comme une capacité d'action efficace dans une situation et un contexte donné, grâce à la mobilisation des connaissances acquises et à la capacité de les mobiliser à bon escient en temps opportun.

47. Quatre registres principaux : - devenir automobiliste (vivre dans et avec l'automobile, vivre avec les autres) ; - gérer son déplacement (maîtriser le véhicule, s'adapter aux situations...) ; - les états dégradés du système (anticiper et prévenir les risques, faire face aux risques dans les situations d'urgence...); - comprendre le phénomène automobile (aspects physiologiques, psychologiques et sociologiques...). 
48. Par exemple, les forces physiques, telles l'énergie cinétique et la force centrifuge, doivent être expérimentées sur le terrain.

49. L'Apprentissage Anticipée de la conduite permet d'apprendre à conduire dès l'âge de 16 ans. Elle se décompose en trois phases : la première comprend la formation initiale, qui est dispensée par un moniteur diplômé, travaillant au sein d'une auto-école; la deuxième, est une phase de conduite accompagnée, supervisée par les parents; celle-ci s'étale sur une période de 1 à 3 ans pendant laquelle l'apprenti-conducteur doit faire au moins $3000 \mathrm{~km}$; la troisième phase fait l'objet d'un suivi pédagogique réunissant l'accompagnateur, l'apprenti et l'enseignant de la conduite, durant la phase accompagnée.

50. Le conducteur à l'impression de pouvoir contrôler le risque et y faire face mieux que les autres ; mais ce n'est qu'une illusion.

51. Trois types:

- la sécurité primaire: la recherche ayant pour objectif la prévention contre l'accident, analyse les causes techniques et comportementales ayant entrainé l'accident.

- La sécurité secondaire : la recherche est axée sur la protection des occupants, et des usagers de la route (piétons, deux roues) afin de diminuer les conséquences de l'accident (suppression des accessoires dangereux comme les gouttières, rétroviseur escamotables...).

- La sécurité tertiaire : la recherche est axée sur l'analyse des accidents pour gérer l'aprèsaccident (alerte et secours)

52. Commandé par le gouvernement.

53. La matrice Goal Driver Education (GDE).

54. Les projets de vie et les tendances personnelles (style/condition de vie, motivation, maîtrise de soi, valeurs personnelles) influencent le comportement au volant.

55. Impact des objectifs du déplacement sur la conduite, effet de la pression sociale à l'intérieur du véhicule...

56. Règles de circulation, adaptation de la vitesse, communication...

57. Contrôle de la direction et de la position, phénomènes physiques...

58. Pédagogie initiée par Tyler (1935) qui propose une organisation scientifique et rationnelle de l'éducation, à l'instar de l'organisation scientifique du travail théorisée par Taylor en 1911.

59. Emprunté au dérivé grec autonomia ce terme concerne en 1815 la philosophie kantienne, l'autonomie de la volonté, d'où ressort une valeur psychologique: liberté, indépendance, lesquelles sont des valeurs rattachées à la fois à l'homme et à la représentation symbolique de la voiture.

60. Depuis les pionniers de l'automobile, l'image mentale de la voiture s'articule autour de la notion de performance.

\section{RÉSUMÉS}

Avec l'évolution des techniques et des technologies appliquées à l'automobile l'environnement routier est devenu un espace complexe, dans lequel la règlementation et le permis de conduire, se révèlent insuffisants pour garantir la sécurité des usagers de la route. La formation du conducteur ne serait-elle plus en adéquation avec la complexité du système routier? Des recherches ont été menées sur le plan européen, afin d'analyser les effets des systèmes de formation du conducteur sur le comportement et leurs capacités (ainsi que leurs limites) à ouvrir 
des perspectives. À la lumière de ces recherches, la capacité auto-évaluative a été retenue comme dynamique de changement dans les attitudes et comportement du conducteur.

With the development of techniques and technologies applied to the automobile, the road environment became a complex area in which the regulation legislation and driving license prove insufficient to ensure the safety of all road users. Would driver's training more in line with the complexity of the road system? Research was conducted at European level, in order to analyze the effects of the driver on the behavior and abilities training systems (and their limits) opportunities. In light of this research, the self-evaluative capacity was selected as a dynamic change in the attitudes and behavior of the driver.

\section{INDEX}

Mots-clés : conduite, comportement, conducteur, apprentissage, permis de conduire, risques routiers, auto-évaluation, pensée réflexive

Keywords : driving, behavior, driver, learning, driving license, road hazards, self-assessment, reflexive thinking

\section{AUTEURS}

\section{LINE COURTEAUD}

Doctorante en sciences de l'éducation, Aix-Marseille Université, EA 4671 ADEF, ENS de Lyon, IFE

\section{CHRISTIAN ROUX}

Professeur en sciences de l'éducation, Aix-Marseille Université, EA 4671 ADEF, ENS de Lyon, IFE 